Volume 2

Issue 4 -- Integrative Medicine

Article 3

$11-20-2015$

\title{
Small Intestinal Bacterial Overgrowth: A Case-Based Review
}

Kristen H. Reynolds

Follow this and additional works at: https://aah.org/jpcrr

Part of the Integrative Medicine Commons, and the Primary Care Commons

\section{Recommended Citation}

Reynolds KH. Small intestinal bacterial overgrowth: a case-based review. J Patient Cent Res Rev. 2015;2:165-173. doi: 10.17294/2330-0698.1209

Published quarterly by Midwest-based health system Advocate Aurora Health and indexed in PubMed Central, the Journal of Patient-Centered Research and Reviews (JPCRR) is an open access, peer-reviewed medical journal focused on disseminating scholarly works devoted to improving patient-centered care practices, health outcomes, and the patient experience. 


\title{
Small Intestinal Bacterial Overgrowth: A Case-Based Review
}

\author{
Kristen H. Reynolds, MD
}

Aurora Wiselives Center and Aurora UW Medical Group, Aurora Health Care, Milwaukee, WI

\begin{abstract}
Small intestinal bacterial overgrowth (SIBO) is a condition of increased microbial load in the small intestine. The microbes feed on dietary carbohydrates and starches via fermentation, leading to gas production, inflammation and damage to the lining of the small intestine. Clinical presentation is varied, including abdominal pain, bloating, malabsorption and systemic symptoms. SIBO is associated with many challenging and chronic conditions such as fibromyalgia, chronic fatigue and chronic pain syndromes, and has been shown to be a causative factor in two out of three cases of irritable bowel syndrome. Symptoms improve with antimicrobial treatment, but recurrence is common. Many providers may not be aware of SIBO. This narrative review highlights a clinical case and the most recent literature regarding SIBO, including history, clinical presentation, prevalence, pathophysiology, diagnostic workup, treatment and prevention. Integrative medicine approaches, including diet, supplements and manual therapies, are also reviewed. SIBO can be a challenging condition and requires an integrative, patientcentered approach. Further studies are needed to guide clinicians in the workup and treatment of SIBO. (J Patient-Centered Res Rev. 2015;2:165-173.)
\end{abstract}

Keywords small intestinal bacterial overgrowth, SIBO, irritable bowel syndrome, IBS

Our understanding of the important role of gut bacteria (the microbiome) in health and disease is rapidly evolving. This narrative review highlights small intestinal bacterial overgrowth (SIBO, pronounced "see-boe"), a condition of increased microbial load in the small intestine. The microbes feed on dietary carbohydrates and starches via fermentation, leading to gas production, inflammation and subsequent damage to the lining of the small intestine. Clinical presentation is varied and can include abdominal pain, bloating, malabsorption and systemic symptoms. ${ }^{1}$ This article will review the history, epidemiology, pathophysiology, clinical presentation, diagnosis, treatment and controversies of this underrecognized and often-challenging condition.

\section{Illustrative Case}

A previously thriving 8-year-old girl presented with onset of sore throat, fever and abdominal pain. Streptococcal pharyngitis was ruled out and the

Correspondence: Kristen H. Reynolds, MD, Aurora Wiselives Center, 8320 W. Bluemound Road, Suite 125, Wauwatosa, WI, 53213, T: 414-302-3800, F: 414-302-3813, Email: kristen.reynolds@aurora.org diagnosis of viral pharyngitis made. Within a few days the fever and sore throat resolved, but the abdominal pain remained and severe insomnia ensued. Additional workup during the following two months included a normal abdominal ultrasound and extensive blood work, with the only abnormal results being elevated bilirubin, thought secondary to Gilbert's syndrome and confirmed by a gastroenterologist. The mother (and author) pursued lactulose breath testing (LBT). Results showed a rise in hydrogen consistent with SIBO. The child was treated with a 2 -week course of rifaximin $200 \mathrm{mg}$ by mouth 3 times a day, with resolution of abdominal pain and insomnia within 2 days of treatment. Repeat breath testing was not performed, as clinical symptoms had improved.

Six months later the child again developed abdominal pain and insomnia with a similar viral pharyngitis trigger, and repeat LBT confirmed recurrent SIBO. Clinical symptoms did not improve with a repeat course of rifaximin. After four months of ongoing symptoms, multiple weeks of missed school, weight loss and guaiac-positive stool, esophagogastroduodenoscopy and colonoscopy were performed, remarkable for only "mild inflammation" on gastric biopsy. Disaccharidase testing was normal. Further review of the breath test 
revealed elevations in both methane and hydrogen (elevated methane requires combination antimicrobial therapy). Given the child's young age, parents chose to avoid neomycin and pursue herbal treatment. The child's insomnia and abdominal pain resolved within 1 week of a 4-week course of neem (an Indian herb) and allicin (the active component in garlic), an herbal treatment for SIBO.

\section{History}

SIBO was first described in the literature in 1939 by Barber and Hummel, who observed the development of macrocytic anemia in patients with intestinal strictures. ${ }^{2}$ In the mid-20th century, Sidney Hass developed the Specific Carbohydrate Diet and had much success treating children with celiac disease and other gastrointestinal disorders with this diet. Shortly after he published his book, The Management of Celiac Disease, in 1951, the diet fell out of favor as gluten was recognized as the culprit in celiac disease (the Specific Carbohydrate Diet eliminates all grains). Dr. Hass' work was furthered by Elaine Gottschall, author of Breaking the Vicious Cycle, and Natasha Campbell-McBride, author of the book and diet Gut and Psychology Syndrome. These diets are currently used in the treatment and prevention of SIBO as they are low in fermentable starches. ${ }^{3}$

In 2000, Pimentel and his team at Cedars-Sinai Medical Center published a report showing that SIBO was present in $78 \%$ of patients with irritable bowel syndrome (IBS), and that treatment with antibiotics improved symptoms. ${ }^{4}$ Since then numerous studies have supported bacterial overgrowth as a cause for IBS, ${ }^{5}$ and in 2014 Pyleris et al. showed that $67 \%$ of patients with IBS have SIBO as evidenced by duodenal aspiration and culture. ${ }^{6}$ Once a bacterial basis for IBS was established, the nonabsorbable antibiotic rifaximin was shown to improve IBS with just one course. ${ }^{7}$ In May 2015 the U.S. Food and Drug Administration (FDA) approved rifaximin for the treatment of diarrhea-predominant IBS. Many experts consider IBS and SIBO to be one in the same.

IBS affects up to $11.2 \%$ of the population ${ }^{8}$ and is a challenging condition to manage and treat. Bacterial overgrowth is a piece of the IBS puzzle that until now has been underrecognized. Just as ulcers were thought to be due to stress and it took over a decade for clinicians to accept Helicobacter pylori as the primary etiology,
SIBO also has been dismissed by much of the medical community. New research is bringing this condition to light.

\section{Epidemiology}

The prevalence of SIBO in the general population is unknown but estimated to be $0-35 \%$ in healthy individuals. ${ }^{9}$ Anywhere from $30 \%$ to $85 \%$ of adult patients with IBS are estimated to have SIBO, ${ }^{9-11}$ with the most current data reporting $67 \%$ as determined by duodenal aspiration and culture. ${ }^{6}$ Two meta-analyses have shown 3.5-9.6-fold increased odds of SIBO in patients with IBS. ${ }^{12}$ In the United States and Europe, one in five school-aged children have been diagnosed with abdominal pain-related functional gastrointestinal disorders, including IBS and functional abdominal pain; SIBO has been shown to occur in $34 \%$ of pediatric IBS patients. ${ }^{10}$ A 2015 study demonstrated that $63 \%$ of children aged 4-17 years who were hospitalized for abdominal pain had SIBO. ${ }^{13}$

Elderly patients may be particularly susceptible to SIBO due to a lack of gastric acid and the use of medications that slow gastrointestinal transit. ${ }^{9}$ SIBO prevalence may be as high as $15 \%$ in the elderly and is an important cause of unexplained diarrhea in this population. ${ }^{12,14}$ SIBO also is common in patients with liver cirrhosis $(50 \%)$, celiac disease $(50 \%)^{9}$ and gastroparesis $(39 \%){ }^{15}$

\section{Pathophysiology}

There are several physiologic mechanisms in place to prevent bacterial overgrowth in the small intestine. These include gastric acid, the migrating motor complex (MMC, a brush-like wave that washes the small bowel during fasting), the intestinal mucosa, the gut-immune system, enzymes, commensal bacteria and the physical barrier of the ileocecal valve. ${ }^{2}$ Anything disrupting these mechanisms can increase the risk of SIBO.$^{14}$

The MMC is a cyclic, recurring motility pattern that occurs in the stomach and small bowel between meals. Regulation of the MMC is complex and involves various hormones and activation of the enteric and parasympathetic nervous systems. Phase III of the MMC, the most active of its four phases, has been shown to be absent or decreased in SIBO, IBS and functional dyspepsia, and can be induced by motilin, erythromycin and serotonin. Vagotomy abolishes part of the MMC. Nerve damage to the MMC plays a part 
in diabetic gastroparesis, and erythromycin is a wellknown treatment that works by restoring the gastric MMC and enhancing gastric motility. ${ }^{16}$

Diabetic enteropathy, gastric bypass, pseudo-obstruction, adhesions, narcotics and Ehlers-Danlos syndrome are known risk factors that slow gastrointestinal transit, and thus predispose individuals to SIBO. The association of proton pump inhibitors with SIBO had been conflicting until a 2013 meta-analysis showed an odds ratio for SIBO of 2.282 (95\% confidence interval [CI]: 1.238-4.205) in those who used proton pump inhibitors compared to those who did not. ${ }^{1}$ Table 1 lists various conditions that can predispose one to developing SIBO. See Table 2 for factors that protect against the development of SIBO and Table 3 for factors that affect the microbiome and may influence SIBO development.

Infectious gastroenteritis can lead to SIBO and IBS through damage to the enteric nerves of the MMC;

Table 1. Conditions that predispose toward development of small intestinal bacterial overgrowth

\begin{tabular}{l}
\hline Achlorhydria (surgical, iatrogenic, autoimmune) \\
Motor abnormalities \\
Scleroderma \\
Intestinal pseudo-obstruction \\
Diabetic enteropathy \\
Vagotomy \\
Abnormal communication between colon and small bowel \\
Fistulas between colon and small bowel \\
Resection of ileocecal valve \\
Structural abnormalities \\
Systemic and intestinal immune deficiency states \\
Surgical loops (billroth ii, entero-entero anastomosis, \\
Rou-en-Y) \\
Duodenal or jejunal diverticula \\
Partial obstruction of small bowel (stricture, adhesions, \\
tumors) \\
Large small-intestinal diverticulosis \\
Systemic diseases (celiac, cirrhosis, pancreatic exocrine \\
insufficiency, nonalcoholic fatty liver) \\
Alcoholism
\end{tabular}

Reprinted from Chedid et al. Herbal therapy is equivalent to rifaximin for the treatment of small intestinal bacterial overgrowth. Glob Adv Health Med. 2014;3(3):16-24, with permission from Global Advances in Health and Medicine LLC.
$25 \%$ of patients with acute gastroenteritis will develop postinfectious IBS. ${ }^{17}$ Studies have shown that the bacteria involved in infectious gastroenteritis, specifically Campylobacter jejuni, Salmonella, Escherichia coli and Shigella, produce cytolethal distending toxin (Cdt). Cdt antibodies cross-react with vinculin, a key protein in the tight junctions lining the gut and a player in nerve cell motility, through molecular mimicry. This alters the enteric nervous system, thus affecting motility and predisposing to IBS and SIBO. Blood biomarkers to anti-Cdt and anti-vinculin are elevated in diarrhea-predominant IBS, and may be helpful in distinguishing this from inflammatory bowel disease in the workup of chronic diarrhea. ${ }^{18}$

The organisms accounting for overgrowth in SIBO are mainly gram-negative aerobes and anaerobic species, including Escherichia coli, Enterococcus spp., Klebsiella pneumonia and Proteus mirabilis. ${ }^{9}$ Another class of microbes involved with SIBO includes methanogens, primitive anaerobes that belong to the kingdom Archaea. Methanogens feed off of hydrogen and produce methane. Methanobrevibacter smithii is the dominant methanogen in the intestine. These organisms are difficult to cultivate and are resistant to many common antibiotics. ${ }^{19}$

Methane was previously thought to be an inert gas, but current evidence suggests it acts like a neuromuscular transmitter to slow intestinal transit. ${ }^{19}$ The presence

Table 2. Factors that protect against development of small intestinal bacterial overgrowth

Gastric acid
Pancreatic enzymes
Bile acids
Cholecystectomy
Motility
Migrating motor complex
Biofilm
Secretory immunoglobulin A
Reprinted from Chedid et al. Herbal therapy is equivalent
to rifaximin for the treatment of small intestinal bacterial
overgrowth. Glob Adv Health Med. 2014;3(3):16-24, with
permission from Global Advances in Health and Medicine LLC.


Table 3. Extrinsic factors that alter the gut microbiome and may influence development of small intestinal bacterial overgrowth

FODMAPs $^{*}$ (fructose, lactose, galactans, fructans,
sugar alcohols)
Proton pump inhibitors
Antimotility agents
Fiber
Prebiotics
Probiotics
Antibiotics

${ }^{*} F O D M A P$ is an acronym for a group of highly fermentable foods (fermentable oligo-, di-, and monosaccharides and polyols).

Reprinted from Chedid et al. Herbal therapy is equivalent to rifaximin for the treatment of small intestinal bacterial overgrowth. Glob Adv Health Med. 2014;3(3):16-24, with permission from Global Advances in Health and Medicine LLC.

of methane on breath testing is almost universally associated with constipation-predominant IBS. ${ }^{19-22}$

Imbalances in the gut microbiome known as dysbiosis are a key feature in SIBO. Such imbalances can disrupt epithelial tight junctions, increasing small intestinal permeability, translocation of endotoxins and induction of proinflammatory cytokines. The gut microbiota has a bidirectional interaction with immune function, digestion, metabolism and gut-brain communication. Dysbiosis has been linked to disorders of mood and behavior as well as numerous gastrointestinal and systemic disorders. ${ }^{11}$

\section{Clinical Presentation}

The manifestations of SIBO have been described as "protean," and can range from mild IBS-type symptoms to severe enteropathy, malabsorption, weight loss and malnutrition. ${ }^{11}$ Typical presentation includes abdominal pain, bloating, flatulence, nausea and altered bowel patterns, including diarrhea and/or constipation. ${ }^{2}$ Systemic symptoms are thought to be secondary to cellular injury from enterotoxin production and bacterial adhesion. ${ }^{1}$ Inflammation can be profound. ${ }^{23}$ This can lead to a damaged intestinal lining and malabsorption, resulting in systemic manifestations of anemia, weight loss, neuropathy, osteoporosis and nutritional deficiencies. $^{2}$ In addition to IBS, SIBO is associated with fibromyalgia, chronic fatigue syndrome, restless leg syndrome, rosacea, arthralgias, chronic prostatitis and pelvic pain syndromes, Parkinson's, interstitial cystitis and more. ${ }^{11,24,25}$ Fatigue is the most prevalent symptom in children diagnosed with $\operatorname{SIBO}(75 \%),{ }^{10}$ and was a prominent feature in the case described.

\section{Diagnosis}

SIBO has conventionally been defined as an excessive concentration of bacteria in the small intestine based on culture of a jejunal aspirate. This approach is limited in clinical practice by high cost, invasive nature, lack of standardization, sampling error and an inability to reach the distal small bowel. ${ }^{2}$ More recently, SIBO diagnosis has been defined as "measurable changes in exhaled gases produced by the metabolism of orally ingested carbohydrates," otherwise known as breath testing. ${ }^{2}$ Breath testing is more readily available, safe, inexpensive and noninvasive, and may be more inclusive when lactulose is used as the substrate as it is more likely to include cases of distal SIBO. There was staunch criticism of the breath test after it started being used for IBS, though it was used without question for SIBO prior to that. ${ }^{25}$ With recent studies confirming bacterial overgrowth as an underlying etiology for IBS, along with the challenges of small bowel aspiration, breath testing is regaining momentum as a useful diagnostic tool.

Breath testing relies on the principle that bacterial metabolism (fermentation) of nonabsorbed carbohydrates is the sole source of hydrogen and methane gas in exhaled breath. After ingestion of a substrate, hydrogen and methane can be measured by gas chromatography in exhaled breath and reported in parts per million (ppm). Lactulose and glucose are the most frequently used substrates for breath testing. Glucose is a monosaccharide that is completely absorbed in the proximal small intestine. In the presence of SIBO, it is fermented into gases that are absorbed and can be measured via exhaled breath. Since it does not reach the distal small intestine, it may miss cases of distal SIBO. Lactulose is a nonabsorbable disaccharide and has been clinically used as an osmotic laxative. ${ }^{2}$ It passes intact through the normal small intestine to the colon where it is metabolized to short-chain fatty acids and gases that are also absorbed and can be measured 
via exhaled breath; in the presence of SIBO, lactulose is fermented in the small intestine, causing an earlier rise in breath gas. ${ }^{2}$ It is important to measure both hydrogen and methane, as treatments vary based on the gas present.

Lactulose breath testing is the most commonly used procedure for diagnosing SIBO, ${ }^{11,22}$ and QuinTron Instrument Company (Milwaukee, WI) is the analyzer most frequently cited in the literature. ${ }^{21}$ Test preparation requires a 36-hour limitation of slowly-absorbed carbohydrates (such as bread and potatoes) and fiber, as well as a restriction on certain medications. The protocol includes a baseline breath sample followed by oral ingestion of $10 \mathrm{~g}$ of lactulose in $200 \mathrm{ml}$ of water. Subsequent breath samples are collected every 15-20 minutes over a 120-240-minute period. ${ }^{2}$

Evaluating breath test results is difficult due to lack of a reliable and reproducible gold standard test for SIBO and variable accuracy for both glucose breath testing (GBT) and LBT. A 2014 review calculated the sensitivity, specificity and diagnostic accuracy to be $63 \%, 82 \%$ and $72 \%$ for GBT and $53 \%, 86 \%$ and $55 \%$ for LBT, respectively. They concluded GBT to be the most accurate test for diagnosing SIBO, with a caveat that it may miss distal SIBO, as glucose is quickly absorbed in the proximal small intestine. ${ }^{2}$ A more recent study using GBT as the gold standard showed a higher sensitivity $(64 \%)$ for LBT. $^{2}$ Testing for methane in addition to hydrogen improves diagnostic accuracy. ${ }^{2}$ There remains a need for a gold standard test for SIBO. ${ }^{2}$

It is important to view results with the clinical context in mind. If a patient reports symptoms of increased gut motility (loose stools) after ingestion of lactulose, then the substrate may have reached the colon early. This may result in a late spike in methane and/or hydrogen, with otherwise low levels throughout the rest of the test.

Interpretation of hydrogen breath test results is also challenging, as there is no universally recognized or validated standard for a positive study. A baseline (fasting) breath hydrogen level of $\geq 20 \mathrm{ppm}$ or a rise in hydrogen of $\geq 12$ ppm from baseline is considered positive. ${ }^{2,10}$ Many microbes (including methanogens and sulfate-reducing bacteria) utilize hydrogen gas as their energy source, which can impair the accurate detection of hydrogen. ${ }^{5}$
Methane breath testing positivity is variably defined in the literature, ranging from $>1 \mathrm{ppm}$ to $\geq 3-12 \mathrm{ppm}$ at any time during the test. Methane breath testing may show two patterns: a high baseline level and early rise in breath methane associated with SIBO, or a late rise corresponding to the arrival of lactulose at the colon. ${ }^{19}$ Experts in the field typically consider a breath methane $\geq 3 \mathrm{ppm}$ to be positive if constipation is present, and $\geq 12 \mathrm{ppm}$ to be positive if constipation is absent. ${ }^{26}$

\section{Treatment}

The treatment and subsequent prevention of SIBO are directed at eliminating the microbial overgrowth, modifying the diet and addressing motility issues. Antimicrobials for SIBO include both prescription and herbal options.

Prescription Antimicrobials: There are many different antibiotics that have been used for the treatment of SIBO. A 2013 review of SIBO treatment with any antibiotic versus placebo demonstrated an odds ratio of 2.55 (95\% CI: 1.29-5.04) for breath test normalization. ${ }^{12}$ One of the most studied antibiotics for SIBO is rifaximin, with an overall breath test normalization rate of $49.5 \%$ (95\% CI: $44.0-55.1)$ in eight clinical trials. ${ }^{12}$ Rifaximin is a nonabsorbable antibiotic with minimal side effects. It is the antibiotic of choice for $\mathrm{SIBO}^{22}$ and works best in hydrogenpredominant cases. Rifaximin must be dosed at $550 \mathrm{mg}$ orally 3 times a day for 2 weeks, as lower doses and twice-daily dosing are not as efficacious. ${ }^{27}$ Rifaximin has been shown to be safe and effective in the pediatric setting at a dose of $600 \mathrm{mg}$ daily ${ }^{28}$ (the author prefers to divide this into twice daily dosing), and was used with initial success in the illustrative case presented. Insurances should now be covering rifaximin given the recent FDA approval; prior authorization may be needed.

For methane-predominant SIBO, the combination of rifaximin with neomycin (500 $\mathrm{mg}$ orally twice daily) is the most effective therapy for clinical improvement of SIBO and elimination of breath methane in the adult population. ${ }^{19,29}$ Methane on breath testing is almost universally associated with constipation, and the combination of rifaximin and neomycin improves constipation in a manner dependent on eradication of breath methane to $<3 \mathrm{ppm}^{20,30}$ 
Breath test normalization is highly associated with clinical improvement. Further randomized, double-blind controlled trials are needed to clarify best treatment options for SIBO. ${ }^{12}$

Prebiotics and Probiotics: Treatment with antibiotics alone does not fully address the microbial dysbiosis associated with SIBO, as antibiotics do not restore normal flora. Probiotics are thought to enhance gut barrier function, decrease inflammation, stabilize gut flora and potentially modulate visceral hypersensitivity. The addition of pre- or probiotics is an understudied but possible option for the treatment of SIBO. ${ }^{12}$ Some experts currently avoid pre- and probiotics during the initial stages of treatment, as these could theoretically feed the underlying bacterial overgrowth.

Guar gum is a prebiotic agent that favors the growth of Bifidobacteria and Lactobacillus species, among others. Rifaximin combined with partially hydrolyzed guar gum was used in one study with a breath test normalization rate of $85 \%$ (95\% CI: $70.2-94.3) .{ }^{31}$

Herbal Antimicrobials: Antibiotic treatment of SIBO comes with variable success, potential for adverse side effects and unknown long-term consequences on the gut microbiota. Relapse of SIBO, which occurred in the aforementioned illustrative case, is common, with recurrence rates up to $44 \%$ within 9 months after successful eradication. ${ }^{32}$ For these reasons, many patients and clinicians have looked to herbals (plantbased compounds) for the treatment of SIBO. There are many herbals with documented antimicrobial activity. Though commonly used by herbalists, naturopaths and holistic clinicians for years, the use of antimicrobial herbs for SIBO had not been reported in the literature until recently.

A ground-breaking study from Johns Hopkins in 2014 showed that herbal therapies are at least as effective as rifaximin ( $46 \%$ vs. $34 \%$, respectively; $\mathrm{P}=0.24$ ) for resolution of SIBO by LBT, and appear to be as effective as triple antibiotic rescue therapy for rifaximin nonresponders $(57.1 \%$ vs. $60 \%, \mathrm{P}=0.89)$, with fewer adverse effects. ${ }^{11}$ Experts in the field have used peppermint oil, neem, allicin, berberine (goldenseal or Oregon grape), wormwood (Artemisia) and oregano (Origanum). The Johns Hopkins study used a proprietary blend, which is described in the paper and included several of these botanicals. ${ }^{11}$

A number of double-blind, placebo-controlled studies have shown enteric-coated peppermint oil (standard dose $0.2 \mathrm{ml}, 3$ times a day) useful in improving the symptoms of IBS, and a 2002 case report of enteric-coated peppermint oil for SIBO described both laboratory and clinical improvement. ${ }^{33}$ The active component in garlic, allicin, has been shown to have activity against enteric pathogens ${ }^{34}$ and is essential in the herbal treatment of methane-positive SIBO.$^{25,26}$ For additional information on dosing botanicals, see Allison Siebecker's website www.siboinfo.com.

Prokinetics and Diet: Addressing gut motility and modifying the diet are two important aspects of SIBO treatment that are aimed at preventing recurrence. As previously mentioned, the migrating motor complex is thought to be disrupted in the case of SIBO, and agents that activate the MMC are essential for preventing recurrence. The most commonly used agents are low-dose erythromycin, prucalopride and low-dose naltrexone. Other agents such as ginger show promise.

Erythromycin has long been used to stimulate gastrointestinal motility, especially for diabetic gastroparesis. ${ }^{16}$ Historically, tegaserod (a serotonin agonist) was given in an attempt to improve phase III of the MMC. Both medications have been shown to prevent recurrence of SIBO; ${ }^{24,35}$ however, tegaserod has been withdrawn from the U.S. market for safety reasons. Prucalopride (available in Canada and Europe) is a safer alternative to tegaserod but has not been directly studied for SIBO.

Low-dose naltrexone is an opioid antagonist that can stimulate intestinal motility, may have anti-inflammatory properties, and has been used successfully for the prevention of SIBO. It is contraindicated in those taking chronic opioids. ${ }^{24}$ Ginger promotes gastric emptying and motility and modulates serotonin receptors in the enteric nervous system. Ginger has documented efficacy as an antiemetic, ${ }^{36}$ though it has not been well-studied for prevention of SIBO recurrence.

5-hydroxytryptophan (5-HTP) is a precursor to 5-hydroxytryptamine (5-HT, or serotonin). Intravenous 
5-HT has been shown to activate the MMC, though intraluminal administration did not affect motility. ${ }^{16}$ A combination of 5-HTP and ginger is available on the market and has been used successfully in the prevention of SIBO recurrence. ${ }^{26}$ The nine-herb combination product STW 5 (Iberogast $^{\mathbb{R}}$, Steigerwald Arzneimittelwerk GmbH, Darmstadt, Germany) has 5-HT agonist properties, has been extensively studied for IBS and was shown to be a safe and effective option for improving motility. ${ }^{37}$ Though Iberogast is a European product, it is available online.

Additional interventions aimed at preventing recurrence include addressing gastric acid levels (removing acid-blocking medications and supplementing with hydrochloric acid if needed), correcting ileocecal valve dysfunction (through acupuncture, osteopathic manipulation or visceral manipulation), addressing any neurological deficits and treating concomitant diseases that contribute to SIBO. ${ }^{38}$ Seeking and addressing the root cause(s) of SIBO are paramount to successful treatment and prevention of recurrence.

Dietary modification is essential for prevention of recurrence and has a powerful influence on the gut microbiome. Dietary change helps to heal the mucosal lining and also can be used during treatment to alleviate symptoms. Various recommended diets include the Specific Carbohydrate Diet, the Gut and Psychology Syndrome (GAPS) Diet, the Low FODMAP Diet, the Cedars-Sinai Diet, the Paleo Diet or a combination of these. Dr. Siebecker of the SIBO Center for Digestive Health (Portland, OR) has created the SIBO Specific Diet, which is a combination of the Specific Carbohydrate Diet and Low FODMAP Diet. It is even more restrictive, but may offer relief in challenging cases. ${ }^{3}$

A 2-week elemental meal replacement consisting of predigested nutrients in powdered form can be used for treatment, but it is difficult, expensive and may not work as well for methane-producing bacteria. ${ }^{39}$ The idea behind any treatment diet is to starve the bacteria while feeding the patient. Vivonex Plus ${ }^{\circledR}$ (Nestlé HealthCare Nutrition Inc., Florham Park, NJ) is an elemental meal replacement that has been shown to be highly effective in normalizing breath tests and improving clinical symptoms. ${ }^{40}$
FODMAPs (fermentable oligo-, di- and monosaccharides and polyols) — short-chain carbohydrates that are poorly absorbed in the small intestine - are highly fermentable and increase the osmotic load in the intestine, thus increasing luminal distention and symptoms such as visceral hypersensitivity. A 2013 observational study of IBS patients $(13 \%$ of whom had documented SIBO) showed symptomatic improvement on a Low FODMAP Diet. ${ }^{41}$ A subsequent randomized crossover trial provided additional confirmation of the effectiveness of a Low FODMAP Diet in the setting of IBS. ${ }^{42}$

Dietary adherence can be challenging. Consultation with a dietitian, written information and positive breath test results can help patients maintain the diet. ${ }^{41}$

\section{Conclusions}

There is increased recognition of small intestinal bacterial overgrowth as a contributing factor in IBS, functional abdominal pain and a host of other chronic conditions. Though SIBO can be challenging to treat and tends to be recurrent, there are many conventional and integrative treatments that can provide significant relief for patients. Treatment for each individual is unique and must be tailored to the specific clinical condition. Further studies are desperately needed to help clinicians better understand the workup, treatment and prevention of this challenging condition.

\section{Patient-Friendly Recap}

- Small intestinal bacterial overgrowth, or SIBO, is a chronic infection that damages the intestinal lining over time.

- $\mathrm{SIBO}$ is thought to be a causative factor in a majority of cases of irritable bowel syndrome.

- While SIBO symptoms like pain and fatigue can improve with antimicrobial treatment, recurrence is common.

- Integrative medicine approaches, particularly herbal supplements and dietary changes, may provide significant relief for patients with SIBO. 


\section{Acknowledgments}

The author would like to thank Dennis Baumgardner, $\mathrm{MD}$, for his years of ongoing support and guidance, and Brenda Fay, MLIS, and Elisa Sibinski, BS, for their kind and generous assistance with references.

\section{Conflicts of Interest}

None.

\section{References}

1. Lo WK, Chan WW. Proton pump inhibitor use and the risk of small intestinal bacterial overgrowth: a meta-analysis. Clin Gastroenterol Hepatol. 2013;11:483-90. CrossRef

2. Saad RJ, Chey WD. Breath testing for small intestinal bacterial overgrowth: maximizing test accuracy. Clin Gastroenterol Hepatol. 2014;12:1964-72. CrossRef

3. Siebecker A. Small intestine bacterial overgrowth: dietary treatments. http://www.siboinfo.com/diet.html. Accessed September 22, 2015.

4. Pimentel M, Chow EJ, Lin HC. Eradication of small intestinal bacterial overgrowth reduces symptoms of irritable bowel syndrome. Am J Gastroenterol. 2000;95:3503-6. CrossRef

5. Shah ED, Basseri RJ, Chong K, Pimentel M. Abnormal breath testing in IBS: a meta-analysis. Dig Dis Sci. 2010;55:2441-9. CrossRef

6. Pyleris E, Giamarellos-Bourboulis EJ, Tzivras D, Koussoulas $\mathrm{V}$, Barbatzas C, Pimentel M. The prevalence of overgrowth by aerobic bacteria in the small intestine by small bowel culture: relationship with irritable bowel syndrome. Dig Dis Sci. 2012;57:1321-9. CrossRef

7. Pimentel M, Park S, Mirocha J, Kane SV, Kong Y. The effect of a nonabsorbed oral antibiotic (rifaximin) on the symptoms of the irritable bowel syndrome: a randomized trial. Ann Intern Med. 2006;145:557-63. CrossRef

8. Lovell RM, Ford AC. Global prevalence of and risk factors for irritable bowel syndrome: a meta-analysis. Clin Gastroenterol Hepatol. 2012;10:712-21. CrossRef

9. Sachdev AH, Pimental M. Gastrointestinal bacterial overgrowth: pathogenesis and clinical significance. Ther $A d v$ Chronic Dis. 2013;4:223-31. CrossRef

10. Korterink JJ, Benninga MA, van Wering HM, DeckersKocken JM. Glucose hydrogen breath test for small intestinal bacterial overgrowth in children with abdominal pain-related functional gastrointestinal disorders. J Pediatr Gastroenterol Nutr. 2015;60: 498-502. CrossRef

11. Chedid V, Dhalla S, Clarke JO, et al. Herbal therapy is equivalent to rifaximin for the treatment of small intestinal bacterial overgrowth. Glob Adv Health Med. 2014;3:16-24. CrossRef

12. Shah SC, Day LW, Somsouk M, Sewell JL. Meta-analysis: antibiotic therapy for small intestinal bacterial overgrowth. Aliment Pharmacol Ther. 2013;38:925-34. CrossRef

13. Siniewicz-Luzeńczyk K, Bik-Gawin A, Zeman K, Bąk-Romaniszyn L. Small intestinal bacterial overgrowth syndrome in children. Prz Gastroenterol. 2015;10:28-32. CrossRef

14. Quigley EM. Small intestinal bacterial overgrowth: what it is and what it is not. Curr Opin Gastroenterol. 2014;30:141-6. $\underline{\text { CrossRef }}$
15. George NS, Sankineni A, Parkman HP. Small intestinal bacterial overgrowth in gastroparesis. Dig Dis Sci. 2014;59:645-52. CrossRef

16. Deloose E, Janssen P, Depoortere I, Tack J. The migrating motor complex: control mechanisms and its role in health and disease. Nat Rev Gastroenterol Hepatol. 2012;9:271-85. CrossRef

17. Neal KR, Hebden J, Spiller R. Prevalence of gastrointestinal symptoms six months after bacterial gastroenteritis and risk factors for development of the irritable bowel syndrome: postal survey of patients. BMJ. 1997;314:779-82. CrossRef

18. Pimentel M, Morales W, Rezaie A, et al. Development and validation of a biomarker for diarrhea-predominant irritable bowel syndrome in human subjects. PloS One. 2015;10:e0126438. CrossRef

19. Triantafyllou K, Chang C, Pimentel M. Methanogens, methane and gastrointestinal motility. J Neurogastroenterol Motil. 2014;20:31-40. CrossRef

20. Pimentel M, Chatterjee S, Chow EJ, Park S, Kong Y. Neomycin improves constipation-predominant irritable bowel syndrome in a fashion that is dependent on the presence of methane gas: subanalysis of a double-blind randomized controlled study. Dig Dis Sci. 2006;51:1297301. CrossRef

21. Chatterjee S, Park S, Low K, Kong Y, Pimental M. The degree of breath methane production in IBS correlates with the severity of constipation. Am J Gastroenterol. 2007;102: 837-41. CrossRef

22. Pimentel M, Chow EJ, Lin HC. Normalization of lactulose breath testing correlates with symptom improvement in irritable bowel syndrome: a double-blind, randomized, placebo-controlled study. Am J Gastroenterol. 2003;98:412-9. CrossRef

23. Lin HC. Small intestinal bacterial overgrowth: a framework for understanding irritable bowel syndrome. JAMA. 2004;292:852-8. CrossRef

24. Turnbull LK, Mullin GE, Weinstock, LB. Principles of integrative gastroenterology: systemic signs of underlying digestive dysfunction and disease. In: Mullin GE (ed). Integrative Gastroenterology. New York, NY: Oxford University Press, 2011, pp. 89-98.

25. National College of Natural Medicine Continuing Education Online. 2014 SIBO Symposium. http://cemoodle.ncnm.edu/ course/category.php?id=130. Accessed September 22, 2015.

26. National College of Natural Medicine Continuing Education Online. 2015 SIBO Symposium. https://cemoodle.ncnm.edu/ course/search.php?search=SIBOSymposium +2015 . Accessed September 22, 2015.

27. Scarpellini E, Gabrielli M, Lauritano CE, et al. High dosage rifaximin for the treatment of small intestinal bacterial overgrowth. Aliment Pharmacol Ther. 2007;25:781-6. CrossRef

28. Scarpellini E, Giorgio V, Gabrielli M, et al. Rifaximin treatment for small intestinal bacterial overgrowth in children with irritable bowel syndrome. Eur Rev Med Pharmacol Sci. 2013;17:1314-20.

29. Low K, Hwang L, Hua J, Zhu A, Morales W, Pimentel M. A combination of rifaximin and neomycin is most effective in treating irritable bowel syndrome patients with methane on lactulose breath test. J Clin Gastroenterol. 2010;44:547-50. CrossRef 
30. Pimentel M. Rifaximin and neomycin improves constipation. [Comprehensive reports on the latest advances in gastroenterology and hepatology from American College of Gastroenterology 2013 Annual Scientific Meeting]. Gastroenterol Hepatol. 2014;10(Abstract P1689):1-22.

31. Furnari M, Parodi A, Gemignani L, et al. Clinical trial: the combination of rifaximin with partially hydrolyzed guar gum is more effective than rifaximin alone in eradicating small intestinal bacterial overgrowth. Aliment Pharmacol Ther. 2010;32:1000-6. CrossRef

32. Lauritano EC, Gabrielli M, Scarpellini E, et al. Small intestinal bacterial overgrowth recurrence after antibiotic therapy. Am J Gastroenterol. 2008;103:2031-5. CrossRef

33. Logan AC, Beaulne TM. The treatment of small intestinal bacterial overgrowth with enteric-coated peppermint oil: a case report. Altern Med Rev. 2002;7:410-7.

34. Ross ZM, O'Gara EA, Hill DJ, Sleightholme HV, Maslin DJ. Antimicrobial properties of garlic oil against human enteric bacteria: evaluation of methodologies and comparisons with garlic oil sulfides and garlic powder. Appl Environ Microbiol. 2001;67:475-80. $\underline{\text { CrossRef }}$

35. Pimentel M, Morales W, Lezcano S, Sun-Chuan D, Low K, Yang J. Low-dose nocturnal tegaserod or erythromycin delays symptom recurrence after treatment of irritable bowel syndrome based on presumed bacterial overgrowth. Gastroenterol Hepatol (N.Y.). 2009;5:435-42.
36. Ernst E, Pittler MH. Efficacy of ginger for nausea and vomiting: a systematic review of randomized clinical trials. Br J Anaesth. 2000;84:367-71. CrossRef

37. Ottillinger B, Storr M, Malfertheiner P, Allescher HD. STW 5 (Iberogast $^{\mathbb{R}}$ )-a safe and effective standard in the treatment of functional gastrointestinal disorders. Wien Med Wochenschr. 2013;163:65-72. CrossRef

38. Siebecker A. Small intestine bacterial overgrowth: prevention. http://www.siboinfo.com/prevention.html. Accessed September 22, 2015.

39. Siebecker A. Small intestine bacterial overgrowth: elemental diet, drawbacks. http://www.siboinfo.com/elemental-formula. html. Accessed September 22, 2015.

40. Pimentel M, Constantino T, Kong Y, Bajwa M, Rezaei A, Park S. A 14-day elemental diet is highly effective in normalizing the lactulose breath test. Dig Dis Sci. 2004;49:73-7. CrossRef

41. de Roest RH, Dobbs BR, Chapman BA, et al. The low FODMAP diet improves gastrointestinal symptoms in patients with irritable bowel syndrome: a prospective study. Int J Clin Pract. 2013;67:895-903. CrossRef

42. Halmos EP, Power VA, Shepherd SJ, Gibson PR, Muir JG. A diet low in FODMAPs reduces symptoms of irritable bowel syndrome. Gastroenterology. 2014;146:67-75.e5. CrossRef

(C) 2015 Aurora Health Care, Inc. 